\title{
The association between insomnia and cardiovascular diseases
}

This article was published in the following Dove Press journal:

Nature and Science of Sleep

2 May 2010

Number of times this article has been viewed

\section{Kai Spiegelhalder \\ Cathy Scholtes \\ Dieter Riemann \\ Department of Psychiatry and Psychotherapy, University of Freiburg Medical Center, Freiburg, Germany}

Correspondence: Kai Spiegelhalder University of Freiburg Medical Center, Department of Psychiatry and Psychotherapy, Hauptstrasse 5,79I04 Freiburg, Germany

$\mathrm{Tel}+4976 \mid 2706589$

Fax +4976I 2706619

Email kai.spiegelhalder@uniklinikfreiburg.de

\begin{abstract}
Insomnia, the most common sleep complaint in the general population, is defined by difficulty initiating or maintaining sleep, or nonrestorative sleep, accompanied by some form of daytime impairment. In the current review, we present an overview of recent studies on the association between insomnia and cardiovascular disease. It can be concluded that there is growing evidence for the hypothesis that insomnia is associated with an increased risk for cardiovascular disease independently of classic coronary risk factors. Furthermore, insomnia is likely to be associated with hypertension and elevated resting heart rate, both known to lead to cardiovascular disease. However, the existing evidence is not totally consistent and most findings have not been replicated unequivocally. The major limitations of the cited studies include the failure to use state-of-the-art criteria for insomnia diagnosis, the failure to control for depression, and the use of hypnotic medication and sleep apnea as potential confounders. However, the results suggest that insomnia is associated with an increased risk for cardiovascular disease mediated by hypertension or elevated resting heart rate. Consequently, more effort should be dedicated to cope with the high prevalence of insomnia in the general population.
\end{abstract}

Keywords: cardiovascular mortality, hypertension, heart rate, heart rate variability

\section{Introduction}

Insomnia is a broadly used term that is defined by difficulty initiating sleep (DIS), difficulty maintaining sleep (DMS), or nonrestorative sleep, accompanied by some form of daytime impairment (eg, fatigue). ${ }^{1}$ It is the most common sleep complaint in the general population, with a prevalence of approximately $10 \% .^{2}$ Insomnia is often secondary to medical conditions such as cancer, pain, congestive heart failure, depression, anxiety disorders, ${ }^{3}$ or secondary to substance intake (for example caffeine), ${ }^{4}$ but can also be an independent condition, ie, primary insomnia. ${ }^{5}$ Predictors of insomnia include female sex,${ }^{6}$ older age, ${ }^{2}$ low income, and medical or psychiatric conditions. ${ }^{7}$

The current review aims at evaluating existing empirical evidence both from epidemiological and experimental studies supporting the hypothesis that insomnia increases the likelihood for cardiovascular disease and cardiovascular mortality independently of classical coronary risk factors. Possible mediating factors or underlying causes for this relationship will be discussed.

\section{Insomnia and cardiovascular disease/mortality}

In 1999, Schwartz and colleagues published a systematic review on the association between subjectively reported insomnia and cardiovascular disease/cardiovascular 
mortality. ${ }^{8}$ Ten studies were included in this review. All of them revealed a significant or near-significant association between subjective sleep complaints and coronary events. In high quality studies, risk ratios for cardiovascular disease were in the range of 1.5-3.9 in those subjects with prolonged sleep onset latency after adjusting for age and known coronary risk factors. This is comparable to the risk ratios of known cardiovascular risk factors like smoking, hypertension, obesity, and diabetes. However, most of the studies under analysis failed to control for psychotropic medication and depression. Indeed, there is compelling evidence that depression is associated with an increased risk for cardiovascular disease. ${ }^{9,10}$ Furthermore, depression is a predictor for mortality in patients with coronary heart disease, ${ }^{11}$ which might be explained by physical inactivity. ${ }^{12}$ Only a few of the studies that were summarized by Schwartz et $\mathrm{al}^{8}$ adjusted for depression, and in one of them depression removed a large part of the association between insomnia and cardiovascular disease. $^{13}$

More recently, two further longitudinal studies of population-based samples revealed an association between subjective sleep complaints and cardiovascular mortality, at least in men. ${ }^{14,15}$ In the first of these investigations, Nilsson and co-authors investigated over 33,000 participants from Sweden with a mean follow-up period of 17 years in men (aged 12-22 years) and 12 years in women (aged 5-20 years). ${ }^{14}$ Of the men, $21 \%$ (aged $49.6 \pm 7.4$ years) and $31 \%$ of the women (aged $43.7 \pm 6.6$ years) reported DIS and/or DMS. Hypnotic medication was used by $3 \%$ of the men and $5 \%$ of the women. In total, 1,902 males and 397 females died during the follow-up period; 727 males and 85 females due to cardiovascular disease. After adjustment for age and known cardiovascular risk factors (smoking, hypertension, obesity) subjectively reported insomnia was positively associated with cardiovascular mortality. In particular, men with a combination of DIS and DMS had a hazard ratio of 1.71 (confidence interval [CI]: 1.34-2.18) for cardiovascular mortality and women with DIS had a hazard ratio of 1.93 (CI: 1.07-3.47). However, depression was not systematically controlled in this largescale investigation due to a lack of a specific questionnaire or clinical interview to measure psychopathology. Furthermore, hypnotic medication and diabetes were also not included as covariates in the analysis.

Mallon and colleagues found an association between subjectively reported sleep initiation difficulties at baseline and cardiovascular mortality (restricted to coronary artery disease mortality) at a 12-year follow-up. ${ }^{15}$ In this population-based investigation, 1,870 representative subjects from Sweden (aged 45-65 years) were investigated. During the follow-up period, 165 males (18.2\%) and 101 females (10.5\%) died. After adjustment for a wide range of important risk factors (age, smoking, hypertension, obesity, diabetes, marital status, living arrangements, cardiac disease, respiratory disease, gastrointestinal disease, urogenital disease, and depression), DIS was related to cardiovascular mortality in males, but not in females. The adjusted risk ratio for DIS in males was 3.1 (CI: 1.5-6.3). Of note, depression was also assessed in this study, which was found to increase the risk of cardiovascular mortality in males. However, the depression status was evaluated only by a single questionnaire item reducing the validity of this variable. Furthermore, the use of sleeping pills was not assessed.

Although these studies suggest that there is an association between insomnia and cardiovascular mortality, these results are still under debate as more recent investigations failed to confirm the association between insomnia and allcause mortality. ${ }^{16,17}$ In a very large study with more than 1.1 million participants (aged 30-102 years), Kripke and colleagues investigated the impact of subjectively reported sleep duration, subjective complaints of insomnia, and use of prescription sleeping pills on all-cause mortality using a six-year follow-up design. ${ }^{16}$ In this analysis, 29 covariates were entered into the statistical model including cardiovascular risk factors, demographic risk factors, habits, and medication use. Depression was, however, not assessed in this investigation. Concerning sleep duration, a U-shaped association was found with the lowest mortality among those who slept seven hours per night. The hazard ratios for those participants who reported sleeping 10 or more hours per night was 1.41 (CI: 1.34-1.50) in women and 1.34 (CI: 1.28-1.40) in men compared with those who slept seven hours per night. In those who reported sleeping four hours per night, the hazard ratios were 1.11 (CI: 1.01-1.22) in women and 1.17 (CI: 1.06-1.28) in men. Accordingly, the increased risk exceeded $10 \%$ for those reporting very long or very short sleep. However, subjective complaints about insomnia were not independently associated with an increased mortality. As described previously, ${ }^{18}$ the use of prescription sleeping pills was significantly associated with increased mortality independently of sleep duration and complaints of insomnia. When removing other covariates, beyond age and use of prescription sleeping pills, subjective complaints of insomnia had a significant impact on mortality in men with a hazard ratio of 1.24 (CI: 1.17-1.30). Overall, the results of this impressively large investigation indicate that it is not the subjective complaint of insomnia per se 
that is associated with an increased mortality. Instead, short sleep duration and use of prescription sleeping pills, coupled with additional confounding factors, might explain this relationship. However, it is unlikely that insomnia patients will be reassured that their complaints are not associated with an increased mortality risk, but short sleep duration is.

A few years ago, Phillips and Mannino also investigated the impact of insomnia and hypnotic medication on all-cause mortality in 13,500 participants over a follow-up period of $6.3 \pm 1.1$ years. ${ }^{17}$ During this follow-up period, 709 participants died. In the analysis of the association between insomnia and mortality, various covariates including cardiovascular risk factors and depression were controlled. However, in contrast to the investigation of Kripke and colleagues, sleep duration was not included as a possible confounding variable. The results showed that in this sample neither insomnia nor hypnotic medication was significantly associated with an increased mortality after six years. A strength of this investigation is the assessment of depression which was highly associated with insomnia and all-cause mortality, as has been previously shown in other investigations. ${ }^{19}$

In summary, data from the newer epidemiological studies yield inconsistent results. While Nilsson and colleagues ${ }^{14}$ and Mallon and colleagues ${ }^{15}$ found an association between insomnia and mortality, Kripke and colleagues ${ }^{16}$ as well as Phillips and Mannino ${ }^{17}$ did not find this effect. This might be due to several methodological differences. First, Nilsson and colleagues did not control for depression at all, and Mallon and colleagues assessed depression with just a single questionnaire item. Depression, however, is probably an important factor contributing to the association between insomnia and mortality. Considering other possible confounders, the use of hypnotics was not assessed by Nilsson and colleagues and Mallon and colleagues. As Kripke and colleagues found an association between the use of prescription sleeping pills and mortality, this might also explain the results of the Swedish studies. Of note, the study of Kripke and colleagues is the only one in which insomnia and short sleep duration were analyzed as independent variables, which is supported by the relatively low correlation coefficients between these variables. The results suggest that subjectively reported sleep duration is a more important variable for the physician than insomnia complaints when evaluating the risk of death. Considering the study designs, it is important to note that the results of Kripke and colleagues as well as Phillips and Mannino, were based on shorter follow-up periods than the Swedish studies. Accordingly, insomnia might be a risk factor for mortality in the long-term but not in the short-term. Additionally, the studies of Kripke and colleagues as well as Phillips and Mannino, investigated all-cause mortality, not cardiovascular mortality specifically. It is possible that insomnia is a risk factor for cardiovascular disease, but a protecting factor against other diseases associated with mortality. In general, state-of-the-art measures of insomnia, depression, or objectively determined sleep duration including sleep apnea measurement have not been used in any of the epidemiological studies.

\section{Insomnia and hypertension}

Current etiological theories for the development and maintenance of insomnia focus on the role of hyperarousal, a cognitive, emotional, or physiological hyperactivity. ${ }^{20,21}$ Hypertension can be regarded as an indicator of physiological hyperarousal. Consequently, hypertension might be more prevalent in insomnia patients than in good sleepers, which has been tested in several studies over the past 10 years. ${ }^{22-27}$ Some of these studies were prospective in nature investigating whether insomnia is a risk factor for hypertension.

In the first of these, Suka and colleagues investigated the impact of subjectively reported sleep disturbances (either DIS or DMS) on hypertension in 4,794 Japanese male workers (age 40-55 years) with a four-year follow-up period. ${ }^{22}$ Age, smoking, obesity, diabetes, alcohol consumption, and job stress were taken into account as potential confounding factors, however, depression was not assessed. Of the 4,794 participants, 1,483 (30.9\%) developed hypertension (defined as systolic blood pressure $>140 \mathrm{mmHg}$, diastolic blood pressure $>90 \mathrm{mmHg}$, or antihypertensive medication) during the follow-up period. The incidence of hypertension among people with persistent DIS (40.1\%) was significantly higher compared with individuals without DIS (30.6\%). After adjusting for potential confounding factors, persistent DIS was associated with a significantly increased risk for hypertension (odds ratio [OR], 1.96; CI: 1.42-2.70). The incidence of hypertension among persistent DMS was $42.3 \%$. After adjusting for potential confounders, persistent DMS was also significantly associated with an increased risk for hypertension (OR, 1.88; CI: 1.45-2.45).

Focusing on the effects of subjectively reported sleep duration on hypertension, Gangwisch et al conducted a population-based prospective study in 4,810 subjects (aged 32-86 years) with a follow-up assessment period of 8-10 years. ${ }^{23}$ Unfortunately, subjective complaints of insomnia were not assessed in this investigation. Hypertension at follow-up was determined by physician diagnosis, hospital 
diagnosis, or cause of death. After controlling for potential confounding variables, including depression, short sleep duration had a significant predictive value for hypertension. Sleep duration of five hours or less per night was associated with a hazard ratio of 1.76 (CI: 1.37-2.56). This effect was even more pronounced in participants aged 32-59 years (hazard ratio, 2.10; CI: 1.58-2.79). In participants aged 60-86 years at baseline, no association was found between the two variables. According to further analyses, the effect of sleep duration on hypertension is partially mediated by body weight and diabetes.

Phillips and Mannino investigated more than 11,000 subjects (aged 44-64 years) over a six-year follow-up period (6.3 \pm 1.1 years). ${ }^{24}$ The study involved a reanalysis of the data published by the same authors in $2005 .{ }^{17}$ However, in the 2007 analysis, only those participants were included that were free of hypertension or cardiovascular disease at baseline. Hypertension was defined as a systolic blood pressure above $160 \mathrm{mmHg}$, a diastolic blood pressure above $95 \mathrm{mmHg}$, or the use of antihypertensive medication. The analysis was controlled for age, gender, smoking, obesity, diabetes, alcohol consumption, heart disease, respiratory disease, menopausal status, depression, educational level, and income. The combination of DIS, DMS, and nonrestorative sleep was associated with an increased risk of cardiovascular disease (OR, 1.5; CI: 1.1-2.0). A complaint of either DIS or DMS, with or without nonrestorative sleep predicted an increased risk for hypertension (OR, 1.2; CI: 1.03-1.3). However, when restricting the analysis to 1) DIS or DMS with non-restorative sleep or 2) DIS, DMS and nonrestorative sleep, there was no significant association with an increased risk for hypertension. Consequently, the authors of this investigation stated that it is not clear whether the inconsistent effects are of clinical significance.

In another investigation, Phillips and colleagues analyzed data from 1,419 older nonhypertensive individuals (baseline mean age $73.5 \pm 4.4$ years) over a follow-up period of six years..$^{25}$ Hypertension in this study was defined as a systolic blood pressure above $140 \mathrm{mmHg}$, a diastolic blood pressure above $90 \mathrm{mmHg}$, or use of antihypertensive medication. Covariates included smoking, obesity, diabetes, alcohol consumption, heart disease, and pulmonary disease, but not depression. In contrast to previous results, subjectively reported DIS, either alone or in combination with other sleep complaints, predicted a statistically significant reduction of risk for hypertension in men. No association was found in women. Furthermore, subjectively reported DMS was not associated with an altered risk for hypertension.
Using a cross-sectional design, Vgontaz and colleagues investigated the association between hypertension and insomnia as well as polysomnographically determined sleep duration in 1,741 participants from central Pennsylvania (aged $48.7 \pm 13.5$ years). ${ }^{26}$ Insomnia was assessed by a questionnaire requiring duration of the sleep complaint to be more than one year. Polysomnographically determined sleep duration was classified into three categories: 1) more than six hours sleep (ca. $50 \%$ of the sample), 2) five to six hours (ca. $25 \%$ ), and 3) less than five hours (ca. 25\%). Hypertension was defined as a systolic blood pressure above $140 \mathrm{mmHg}$, a diastolic blood pressure above $90 \mathrm{mmHg}$, or use of antihypertensive medication. Age, race, gender, smoking, obesity, diabetes, alcohol consumption, and depression as well as sleep disordered breathing and periodic leg movements during sleep, as determined by polysomnography, were included as covariates in the analyses. Compared to those participants who slept more than six hours and did not complain about insomnia or poor sleep, participants with insomnia and less than five hours sleep duration had an increased association with hypertension (OR, 5.12; CI: 2.22-11.79). Of note, the degree of this association was approximately as high as the one between sleep-disordered breathing and hypertension. Furthermore, those participants with insomnia and an objectively determined sleep duration of five to six hours also had an increased OR of having hypertension (OR, 3.5; CI: 1.6-7.9). In contrast, neither insomnia without polysomnographically determined short sleep (OR, 1.31; CI: 0.70-2.46) nor short sleep duration (less than five hours) without any sleep complaints (OR, 1.13; CI: 0.79-1.62), were significantly associated with hypertension.

Using an experimental approach, Lanfranchi and colleagues reported an elevated systolic blood pressure in a small group of well-described normotensive chronic primary insomnia patients that was evident specifically during the night. ${ }^{27}$ This finding suggests that insomnia patients have an altered 24 hour blood pressure profile. Nighttime systolic blood pressure was $111 \pm 15 \mathrm{mmHg}$ in insomnia patients compared to $102 \pm 12 \mathrm{mmHg}$ in healthy controls. As there was no significant difference in daytime blood pressure, day-to-night dipping in systolic blood pressure was lower in insomnia patients $(-8 \pm 6 \%)$ than in the control group $(-15 \pm 5 \%)$. Furthermore, nighttime systolic blood pressure was positively correlated with electroencephalogy (EEG) beta activity during the night suggesting that sleep-related cortical hyperarousa ${ }^{21}$ might be the underlying factor of the increased nocturnal blood pressure. Of note, sleep apnea patients were excluded in this investigation. 
In summary, the results suggest that insomnia and/or short sleep duration are associated with an increased risk for hypertension in middle-aged individuals, ${ }^{22,23}$ but not in older ones. ${ }^{25}$ The effect is probably more pronounced when insomnia is associated with objectively determined short sleep duration. ${ }^{26}$ Additionally, an altered 24 hour blood pressure profile with an increased nocturnal systolic blood pressure might be evident in insomnia patients. The study of Phillips and Mannino ${ }^{24}$ found no association between insomnia and subsequent hypertension. One reason for this differing result might be that hypertension was defined differently in this investigation, with a systolic blood pressure of $160 \mathrm{mmHg}$ instead of $140 \mathrm{mmHg}$, or a diastolic blood pressure of $95 \mathrm{mmHg}$ instead of $90 \mathrm{mmHg}$. Accordingly, it can be speculated that insomnia is only associated with an increased risk for mild to moderate hypertension. Unfortunately, hypnotic medication was not taken into account in any of the above studies. Depression was assessed by some studies, ${ }^{23,24,26}$ but not by all. ${ }^{22,25}$ Sleep apnea was only determined in the investigation of Vgontaz and colleagues ${ }^{26}$ and excluded in the experimental study of Lanfranchi and colleagues. ${ }^{27}$ Furthermore, while several studies investigated the impact of insomnia on subsequent hypertension, no longitudinal study analyzed the impact of hypertension on insomnia. However, this relationship is of interest for evaluating the results of the cross-sectional study conducted by Vgontaz and colleagues. ${ }^{26}$

\section{Insomnia and resting heart rate/ heart rate variability (HRV)}

Elevated resting heart rate and alterations in heart rate variability (HRV) - more specifically, increased sympathetic activity and decreased parasympathetic activity - are subclinical risk factors for cardiovascular disease and mortality. ${ }^{28,29}$ In line with the hyperarousal model, ${ }^{20,21}$ these risk factors might occur with an increased prevalence in patients with chronic insomnia.

There are a few experimental studies in insomnia patients, in which an elevated resting heart rate has been reported for this condition. ${ }^{30,31}$ However, this finding has not been reported consistently. ${ }^{32-34}$ In the largest study so far, Nilsson and colleagues reported that DIS and DMS are associated with a significant elevation in resting heart rate of 0.9 and $0.6 \mathrm{bpm}$, respectively. ${ }^{14}$ In this investigation, another interesting effect was reported: both subjectively reported DIS/DMS and elevated heart rate increased the risk for all-cause mortality in men. The adjusted hazard ratio in insomniacs, who were in the highest heart rate tertile (>70 bpm), was 2.66 (CI: 2.06-3.44) compared with those participants without DIS or DMS, who were in the lowest heart rate tertile ( $<61 \mathrm{bmp})$. However, it has to be borne in mind that Nilsson and colleagues did not use polysomnographic measurements and based their assessment of sleep disturbance on three questionnaire items without fulfilling the criteria for insomnia diagnosis.

HRV has been investigated in insomnia patients by analyzing polysomnographic recordings including electrocardiographic data. ${ }^{31}$ In this frequently cited study, 12 primary insomnia patients (aged $31.2 \pm 6.8$ years) and 12 healthy controls (aged $29.1 \pm 5.2$ years) were investigated. Spectral analysis of the nocturnal beat-to-beat-intervals ${ }^{35,36}$ revealed a significantly increased low frequency power $(0.05-0.15 \mathrm{~Hz})$ in insomnia patients during sleep and nighttime wakefulness, indicating increased sympathetic activity. Additionally, high frequency power $(0.15-0.50 \mathrm{~Hz})$ was decreased in the patients group indicating reduced parasympathetic activity. These results support the hyperarousal model of insomnia. ${ }^{20,21}$ Furthermore, the data suggests that HRV alterations in insomnia, with an altered sympathovagal balance, might lead to an increased risk for cardiovascular disease.

However, this finding has never been replicated. In a recent similar investigation, Jurysta and colleagues found no difference in nocturnal HRV parameters between insomnia patients and healthy controls. ${ }^{37}$ In this investigation, 14 patients with chronic primary insomnia (aged $42 \pm 12$ years) and 14 healthy controls (aged $41 \pm 10$ years) were investigated using polysomnographic recordings.

In addition to these investigations, there are two studies in which HRV parameters have been investigated in insomnia patients during daytime. ${ }^{34,38}$ Both studies reported no between-group differences. Fang and colleagues investigated 18 insomnia patients without any medical or psychiatric comorbidity (aged $34.2 \pm 14.5$ years) and 21 healthy controls (aged $27.8 \pm 8.7$ years) during paced breathing conditions in the afternoon. ${ }^{38} \mathrm{HRV}$ variables included parameters for sympathetic and parasympathetic activity. The only group comparison that revealed a trend towards significance was the one for the parasympathetic parameter. For this parameter, insomnia patients had lower values than the control group. Varkevisser and colleagues studied 11 patients with chronic primary insomnia (aged $43.8 \pm 8.9$ years) and 13 healthy controls (aged $44.9 \pm 7.7$ years) for 24 hours under sleep deprivation. ${ }^{34}$ The experiment was conducted under carefully controlled constant routine conditions without changes in light exposure or temperature. Furthermore, food intake was standardized. However, although between-group differences 
were in the expected direction, the groups did not differ significantly in heart rate or HRV.

Summarizing these experiments, it is likely that insomnia patients have a slightly increased resting heart rate resulting in a slightly increased risk for cardiovascular disease. Concerning nocturnal HRV, the results are inconsistent. One study showed nocturnal HRV alterations in insomnia patients and one study failed to show any between-group differences. During daytime, there is no evidence for HRV alterations in insomnia. Generally, it should be noted that all studies apart from the one of Nilsson and colleagues included only small sample sizes. However, the experimental studies used polysomnographic recordings resulting in the accurate exclusion of sleep apnea syndrome.

\section{Discussion}

In summary, there is growing evidence that insomnia is associated with cardiovascular disease and cardiovascular risk factors, especially hypertension and elevated resting heart rate. However, to date, the existing evidence is not fully consistent and most findings have not been replicated unequivocally. Large-scale investigations typically failed to use standard diagnostic criteria for insomnia. Instead, one or two questionnaire items have been used to determine sleep onset or sleep maintenance difficulties. Daytime consequences, insomnia duration or comorbid sleep disorders like sleep apnea syndrome have usually been neglected. Additionally, it is important to distinguish between subjective insomnia complaints, subjective sleep duration and if possible to determine, objectively determined sleep duration. The importance of measuring subjective and objective sleep duration was recently highlighted by Feige and colleagues. ${ }^{39}$ Depression is a significant confounder of the association between insomnia and cardiovascular disease. However, some studies did not measure depression. Furthermore, the majority of the other investigations used only a small number of questionnaire items to capture the influence of this important confound. The use of hypnotic medication might be another potential confounding factor mediating the association between insomnia and cardiovascular disease. This is particularly important because long-term use of hypnotic medication has been found to be associated with mortality. ${ }^{18,40,41}$ The reason behind this association is still under debate. Hypnotic medication might be an indicator of underlying somatic or psychiatric disease (see for example Kripke and colleagues) $)^{42}$ as epidemiological analyses can never include all possible confounders. Other suggestions include that hypnotics promote suicidal behavior ${ }^{43}$ or increase cancer risk. ${ }^{44}$ In light of this evidence, randomized controlled trials are urgently needed to investigate the longterm effects of hypnotic medication. Regarding the use of benzodiazepine receptor agonists in insomnia patients, only a handful of studies included follow-up measurement of six months to investigate long-term efficacy. ${ }^{45-47} \mathrm{~A}$ further confounder for the association between insomnia and cardiovascular disease is caffeine intake, which might trigger coronary events. ${ }^{4}$ However, caffeine intake is probably not associated with a generally increased risk for cardiovascular diseases. ${ }^{48}$ As mentioned, sleep apnea syndrome has not been taken into account in most of the above cited studies although there might be an underestimated comorbidity between insomnia and sleep-related breathing disorders. ${ }^{49}$ Sleep apnea syndrome is a known risk factor for cardiovascular disease $\mathrm{e}^{50,51}$ and is more prevalent in those with existing cardiovascular morbidity. ${ }^{52}$

Apart from potential confounding variables, what might be the reason for the association between insomnia and cardiovascular disease? One potential answer is inflammation which is strongly linked to cardiovascular disease. ${ }^{53}$ On the other hand, sleep loss was found to be associated with an increased cellular inflammatory signaling. ${ }^{54}$ Similarly, in insomnia patients, inflammatory markers were found to be increased. ${ }^{55}$ Furthermore, cognitive-behavioral therapy for insomnia has been shown to have an effect on immune functioning. ${ }^{56}$ It might further be speculated that amyloid play a role in the association between insomnia and cardiovascular disease. Amyloid- $\beta$ has just recently been linked to the orexin system and the sleep-wake cycle. ${ }^{57}$

Overall, it is likely that insomnia is associated with an increased risk for cardiovascular disease, perhaps mediated by hypertension or elevated resting heart rate. Consequently, more effort should be invested to challenge the high prevalence of insomnia in the general population.

\section{Disclosures}

The authors report no conflicts of interest in this work.

\section{References}

1. American Academy of Sleep Medicine. The International Classification of Sleep Disorders, (ICSD-2): Diagnostic and Coding Manual. 2nd ed. Westchester, IL: American Academy of Sleep Medicine; 2005.

2. Ohayon MM. Epidemiology of insomnia: what we know and what we still need to learn. Sleep Med Rev. 2002;6(2):97-111.

3. Benca RM, Obermeyer WH, Thisted RA, Gillin JC. Sleep and psychiatric disorders - a meta-analysis. Arch Gen Psychiat. 1992;49(8): 651-668.

4. Riksen NP, Rongen GA, Smits P. Acute and long-term cardiovascular effects of coffee: implications for coronary heart disease. Pharmacol Ther. 2009;121(2):185-191. 
5. Edinger JD, Bonnet MH, Bootzin RR, et al. Derivation of research diagnostic criteria for insomnia: report of an American academy of sleep medicine work group. Sleep. 2004;27(8):1567-1596.

6. Zhang B, Wing YK. Sex differences in insomnia: a meta-analysis. Sleep. 2006;29(1):21-22.

7. Riemann D, Voderholzer U. Primary insomnia: a risk factor to develop depression? J Affect Disord. 2003;76(1-3):255-259.

8. Schwartz S, McDowell Anderson W, Cole SR, Cornoni-Huntley J, Hays JC, Blazer D. Insomnia and heart disease: a review of epidemiologic studies. J Psychosom Res. 1999;47(4):313-333.

9. Wulsin L, Vaillant G, Wells V. A systematic review of the mortality of depression. Psychosom Med. 1999;61(1):6-17.

10. Rozanski A, Blumenthal JA, Kaplan J. Impact of psychological factors on the pathogenesis of cardiovascular disease and implications for therapy. Circulation. 1999;99(16):2192-2217.

11. Frasure-Smith N, Lespérance F, Talajic M. Depression following myocardial infarction. Impact on 6-month survival. JAMA. 1993;270(15): 1819-1825.

12. Whooley MA, de Jonge P, Vittinghoff E. Depressive symptoms, health behaviors, and risk of cardiovascular events in patients with coronary heart disease. JAMA. 2008;300(20):2379-2388.

13. Schwartz SW, Cornoni-Huntley J, Cole SR, Hays JC, Blazer DG, Schocken DD. Are sleep complaints an independent risk factor for myocardial infarction? Ann Epidemiol. 1998;8(6):384-392.

14. Nilsson PM, Nilsson JA, Hedblad B, Berglund G. Sleep disturbance in association with elevated pulse rate for prediction of mortality-consequences of mental strain? J Intern Med. 2001;250(6): 521-529.

15. Mallon L, Broman JE, Hetta J. Sleep complaints predict coronary artery disease mortality in males: a 12-year follow-up study of a middle-aged Swedish population. J Intern Med. 2002;251(3):207-216.

16. Kripke DF, Garfinkel L, Wingard DL, Klauber MR, Marler MR. Mortality associated with sleep duration and insomnia. Arch Gen Psychiatry. 2002;59(2):131-136.

17. Phillips B, Mannino MD. Does insomnia kill? Sleep. 2005;28(8): 965-971.

18. Kripke DF, Klauber MR, Wingard DL, Fell RL, Assmus JD, Garfinkel L. Mortality hazard associated with prescription hypnotics. Biol Psychiatry. 1998;43(9):687-693.

19. Schulz R, Beach SR, Ives DG, Martire LM, Ariyo AA, Kop WJ. Association between depression and mortality in older adults: the Cardiovascular Health Study. Arch Intern Med. 2000;160(12):1761-1768.

20. Riemann D, Spiegelhalder K, Feige B, et al. The hyperarousal model of insomnia: A review of the concept and its evidence. Sleep Med Rev. 2010;14(1):19-31.

21. Perlis ML, Giles DE, Mendelson WB, Bootzin RR, Wyatt JK. Psychophysiological insomnia: the behavioral model and a neurocognitive perspective. J Sleep Res. 1997;6(3):179-188.

22. Suka M, Yoshida K, Sugimori H. Persistent insomnia is a predictor of hypertension in Japanese male workers. J Occup Health. 2003;45(6): 344-350.

23. Gangwisch JE, Heymsfield SB, Boden-Albala B, et al. Short sleep duration as a risk factor for hypertension: analyses of the first National Health and Nutrition Examination Survey. Hypertension. 2006;47(5): 833-839.

24. Phillips B, Mannino DM. Do insomnia complaints cause hypertension or cardiovascular disease? J Clin Sleep Med. 2007;3(5):489-494.

25. Phillips B, Buzkova P, Enright P. Insomnia did not predict incident hypertension in older adults in the Cardiovascular Health Study. Sleep. 2009;32(1):65-72.

26. Vgontaz AN, Liao D, Bixler EO, Chrousos GP, Vela-Bueno A. Insomnia with objective short sleep duration is associated with a high risk for Hypertension. Sleep. 2009;32(4):491-497.

27. Lanfranchi PA, Pennestri MH, Fradette L, Dumont M, Morin CM, Montplaisir J. Night time blood pressure in normotensive subjects with chronic insomnia: implications for cardiovascular risk. Sleep. 2009;32(6):760-766.
28. Fox K, Borer, JS, Camm AJ, et al. Resting heart rate in cardiovascular disease. J Am Coll Cardiol. 2007;50(9):823-830.

29. Lahiri MK, Kannankeril PJ, Goldberger JJ. Assessment of autonomic function in cardiovascular disease: physiological basis and prognostic implications. J Am Coll Cardiol. 2008;51(18):1725-1733.

30. Haynes SN, Adams A, Franzen M. The effects of presleep stress on sleep-onset insomnia. J Abnorm Psychol. 1981;90(6):601-606.

31. Bonnet MH, Arand DL. Heart rate variability in insomniacs and matched normal sleepers. Psychosom Med. 1998;60(5):610-615.

32. Monroe LJ. Psychological and physiological differences between good and poor sleepers. J Abnorm Psychol. 1967;72(3):255-264.

33. Freedman RR, Sattler HL. Physiological and psychological factors in sleep-onset insomnia. J Abnorm Psychol. 1982;91(5):380-389.

34. Varkevisser M, Van Dongen HP, Kerkhof GA. Physiologic indexes in chronic insomnia during a constant routine: evidence for general hyperarousal? Sleep. 2005;28(12):1588-1596.

35. Akselrod S, Gordon D, Ubel FA, Shannon DC, Berger AC, Cohen RJ. Power spectrum analysis of heart rate fluctuation: a quantitative probe of beat-to-beat cardiovascular control. Science. 1981;213(4504):220-222.

36. Malik M. Task Force of the European Society of Cardiology and the North American Society of Pacing and Electrophysiology. Heart rate variability standards of measurement, physiological interpretation, and clinical use. Eur Heart J. 1996;17(3):354-381.

37. Jurysta F, Lanquart JP, Sputaels V, et al. The impact of chronic primary insomnia on the heart rate-EEG variability link. Clin Neurophysiol. 2009;120(6):1054-1060.

38. Fang SC, Huang CJ, Yang TT, Tsai PS. Heart rate variability and daytime functioning in insomniacs and normal sleepers: preliminary results. J Psychosom Res. 2008;65(1):23-30.

39. Feige B, Al-Shajlawi A, Nissen C, et al. Does REM sleep contribute to subjective wake time in primary insomnia? A comparison of polysomnographic and subjective sleep in 100 patients. J Sleep Res. 2008;17(2):180-190.

40. Kripke DF, Simons RN, Garfinkel L, Hammond EC. Long-term use of sleeping pills is associated with a survival risk. Arch Gen Psychiatry. 1979;36(1):103-116.

41. Mallon L, Broman JE, Hetta J. Is usage of hypnotics associated with mortality? Sleep Med. 2009;10(3):279-286

42. Kripke DF. Greater incidence of depression with hypnotic use than with placebo. BMC Psychiatry. 2007;7:42.

43. Neutel CI, Patten SB. Risk of suicide attempts after benzodiazepine and/or antidepressant use. Ann Epidemiol. 1997;7(8):568-574.

44. Kripke DF. Possibility that certain hypnotics might cause cancer in skin. J Sleep Res. 2008;17(3):245-250.

45. Krystal AD, Walsh JK, Laska E, et al. Sustained efficacy of eszopiclone over 6 months of nightly treatment: results of a randomized, doubleblind, placebo-controlled study in adults with chronic insomnia. Sleep. 2003;26(7):793-799.

46. Ancoli-Israel S, Richardson GS, Mangano RM, Jenkins L, Hall P, Jones WS. Long-term use of sedative hypnotics in older patients with insomnia. Sleep Med. 2005;6(2):107-113.

47. Krystal AD, Erman M, Zammit GK, Soubrane C, Roth T. Long-term efficacy and safety of zolpidem extended-release $12.5 \mathrm{mg}$, administered 3 to 7 nights per week for 24 weeks, in patients with chronic primary insomnia: a 6-month, randomized, double-blind, placebo-controlled, parallel group, multicenter study. Sleep. 2008;31(1):79-90.

48. Lopez-Garcia E, van Dam RM, Willett WC, et al. Coffee consumption and coronary heart disease in men and women: a prospective cohort study. Circulation. 2006;113(17):2045-2053.

49. Krakow B, Melendrez D, Ferreira E, et al. Prevalence of insomnia symptoms in patients with sleep-disordered breathing. Chest. 2001;120(6):1923-1929.

50. Shahar E, Whitney CW, Redline S, et al. Sleep-disordered breathing and cardiovascular disease: cross-sectional results of the Sleep Heart Health Study. Am J Respir Crit Care Med. 2001;163(1):19-25. 
51. Peker Y, Hedner J, Norum J, Kraiczi H, Carlson J. Increased incidence of cardiovascular disease in middle-aged men with obstructive sleep apnea: a 7-year follow-up. Am J Respir Crit Care Med. 2002;166(2):159-165.

52. Mills PJ, Dimsdale JE, Natarajan L, Ziegler MG, Maisel A, Greenberg BH. Sleep and health-related quality of life in heart failure. Congest Heart Fail. 2009;15(5):228-233.

53. Willerson JT, Ridker PM. Inflammation as a cardiovascular risk factor. Circulation. 2004;109 Suppl II:2-10.

54. Irwin MR, Wang M, Ribeiro D, et al. Sleep loss activates cellular inflammatory signaling. Biol Psychiatry. 2008;64(6):538-540.
55. Burgos I, Richter L, Klein T, et al. Increased nocturnal interleukin-6 excretion in patients with primary insomnia: a pilot study. Brain Behav Immun. 2006;20(3):246-253.

56. Savard J, Simard S, Ivers H, Morin CM. Randomized study on the efficacy of cognitive-behavioral therapy for insomnia secondary to breast cancer, part II: immunologic effects. J Clin Oncol. 2005;23(25):6097-6106.

57. Kang JE, Lim MM, Bateman RJ, et al. Amyloid- $\beta$ dynamics are regulated by orexin and the sleep-wake cycle. Science. 2009;326(5955):1005-1007.

\section{Publish your work in this journal}

Nature and Science of Sleep is an international, peer-reviewed, open access journal covering all aspects of sleep science and sleep medicine, including the neurophysiology and functions of sleep, the genetics of sleep, sleep and society, biological rhythms, dreaming, sleep disorders and therapy, and strategies to optimize healthy sleep. The journal welcomes

\section{Dovepress}

original research, clinical \& epidemiological studies, reviews \& evaluations, case reports and extended reports. The manuscript management system is completely online and includes a very quick and fair peerreview system, which is all easy to use. Visit http://www.dovepress.com/ testimonials.php to read real quotes from published authors. 